\title{
Statistics on Lattice Walks and q-Lassalle Numbers
}

\author{
Lenny Tevlin
}

Liberal Studies, New York University, New York, N.Y. 10003, U.S.A.

\begin{abstract}
This paper contains two results. First, I propose a $q$-generalization of a certain sequence of positive integers, related to Catalan numbers, introduced by Zeilberger, see Lassalle (2010). These $q$-integers are palindromic polynomials in $q$ with positive integer coefficients. The positivity depends on the positivity of a certain difference of products of $q$-binomial coefficients.

To this end, I introduce a new inversion/major statistics on lattice walks. The difference in $q$-binomial coefficients is then seen as a generating function of weighted walks that remain in the upper half-plane.

Résumé. Cet document contient deux résultats. Tout d'abord, je vous propose un $q$-generalization d'une certaine séquence de nombres entiers positifs, liés à nombres de Catalan, introduites par Zeilberger (Lassalle, 2010). Ces $q$ -integers sont des polynômes palindromiques à $q$ à coefficients entiers positifs. La positivité dépend de la positivité d'une certaine différence de produits de $q$-coefficients binomial.

Pour ce faire, je vous présente une nouvelle inversion/major index sur les chemins du réseau. La différence de $q$ -binomial coefficients est alors considérée comme une fonction de génération de trajets pondérés qui restent dans le demi-plan supérieur.
\end{abstract}

Keywords: lattice walks, statistics on words, $q$-integers

\section{Introduction to Lassalle's Sequences and their $q$-analogs.}

Michel Lassalle [Las12] has discussed two related sequences of numbers $A_{k}$ and $\alpha_{k} .\left\{A_{k}\right\}$ is generated by the following recurrence:

$$
A_{n}=(-1)^{n-1} C_{n}+\sum_{j=1}^{n-1}(-1)^{n-j-1}\left(\begin{array}{c}
2 n-1 \\
2 j-1
\end{array}\right) A_{j} C_{n-j}, \quad A_{1}=1
$$

The second sequence is

$$
\alpha_{n}=\frac{2 A_{n}}{C_{n}}
$$

\footnotetext{
${ }^{\dagger}$ Email: ltevlin@nyu.edu

1365-8050 @ 2015 Discrete Mathematics and Theoretical Computer Science (DMTCS), Nancy, France
} 
He proved that both $A_{n}$ and $\alpha_{n}$ are positive integers and each sequence is increasing (and more). (It turns out that the second sequence is simply related to power sums of zeros of Bessel function $J_{0}(z)$ ). It is intriguing to inquire whether there is a natural $q$-analog of these numbers. It may be generated by a $q$-analog of the above recurrence (with $C_{n}(q)=\frac{1}{[n+1]_{q}}\left[\begin{array}{c}2 n \\ n\end{array}\right]_{q}$, a $q$-Catalan):

$$
A_{n}^{\prime}(q)=(-1)^{n-1} q^{n-1} C_{n}(q)+\sum_{j=1}^{n-1}(-1)^{n-j-1} q^{n-j}\left[\begin{array}{c}
2 n-1 \\
2 j-1
\end{array}\right]_{q} A_{j}^{\prime}(q) C_{n-j}(q)
$$

but a slightly renormalized version looks neater:

$$
\begin{aligned}
A_{n}(q)=(-1)^{n-1} q^{3-2 n} C_{n}(q)+(-1)^{n} q^{3-2 n} & {[2 n-1]_{q} C_{n-1}(q) } \\
& +\sum_{j=2}^{n-1}(-1)^{n-j-1} q^{2 j-2 n}\left[\begin{array}{c}
2 n-1 \\
2 j-1
\end{array}\right]_{q} A_{j}(q) C_{n-j}(q)
\end{aligned}
$$

with $A_{1}(q)=1$. It turns out that $A_{n}(q)$ are monic unimodal palindromic polynomials in $q$ with positive integer coefficients. Here are some examples:

\section{Example 1}

$$
\begin{aligned}
& A_{2}(q)=1 \\
& A_{3}(q)=1+q+q^{2}+q^{3}+q^{4} \\
& A_{4}(q)=1+2 q+3 q^{2}+5 q^{3}+6 q^{4}+7 q^{5}+8 q^{6}+7 q^{7}+6 q^{8}+5 q^{9}+3 q^{10}+2 q^{11}+q^{12} \\
& A_{5}(q)=1+3 q+6 q^{2}+12 q^{3}+19 q^{4}+29 q^{5}+41 q^{6}+54 q^{7}+67 q^{8}+80 q^{9}+89 q^{10}+96 q^{11}+98 q^{12} \\
& +96 q^{13}+89 q^{14}+80 q^{15}+67 q^{16}+54 q^{17}+41 q^{18}+29 q^{19}+19 q^{20}+12 q^{21}+6 q^{22}+3 q^{23}+q^{24}
\end{aligned}
$$

The second Lassalle's sequence $\alpha_{k}$ has the following $q$-analog:

$$
\alpha_{n}=\frac{\left(1+q^{n}\right) A_{n}(q)}{C_{n}(q)}
$$

And each of $\alpha_{n}(q)$ is also a monic unimodal palindromic polynomial in $q$ with positive integer coefficients. Here are examples of $\alpha_{n}(q)$

\section{Example 2}

$$
\begin{aligned}
& \alpha_{1}(q)=1+q \\
& \alpha_{2}(q)=1 \\
& \alpha_{3}(q)=1+q \\
& \alpha_{4}(q)=1+2 q+2 q^{2}+2 q^{3}+q^{4} \\
& \alpha_{5}(q)=1+3 q+5 q^{2}+8 q^{3}+9 q^{4}+9 q^{5}+8 q^{6}+5 q^{7}+3 q^{8}+q^{9}
\end{aligned}
$$


The proof of positivity of $\alpha_{k}$ relies (in addition to certain divisibility properties) on the positivity of

$$
\left(\begin{array}{c}
n \\
k-1
\end{array}\right)\left(\begin{array}{l}
n \\
k
\end{array}\right)-\left(\begin{array}{c}
n \\
k-2
\end{array}\right)\left(\begin{array}{c}
n \\
k+1
\end{array}\right)
$$

and Lassalle used the combinatorial interpretation of this difference of binomial coefficients as a generating function of the number of NSEW walks on a square lattice that start at the origin and finish at $(2 k-n-1,1)$ [GKS92].

Similarly, the positivity of $\alpha_{k}(q)$ requires the positivity of

$$
\left[\begin{array}{c}
n \\
k-1
\end{array}\right]_{q}\left[\begin{array}{c}
n \\
k
\end{array}\right]_{q}-q^{2}\left[\begin{array}{c}
n \\
k-2
\end{array}\right]_{q}\left[\begin{array}{c}
n \\
k+1
\end{array}\right]_{q}
$$

However in this case a combinatorial interpretation has to be developed.

\section{Introduction to $q$-enumeration of Lattice walks}

To understand the positivity of (4) combinatorially, i.e. as a generating function of certain weighted lattice walks, I first interpret the $q$-version of the generating function of all NSEW walks as a generating function of a certain (new) inversion statistics on lattice walks.

The total number of lattice walks from $(0,0)$ to $(c, d)$ of length $n$ is given by [DR84]

$$
\left(\begin{array}{c}
n \\
\frac{1}{2}(n-c+d)
\end{array}\right)\left(\begin{array}{c}
n \\
\frac{1}{2}(n-c-d)
\end{array}\right)
$$

Think of a given walk as a word $w$ composed of letters $N, S, E, W$. Then, the walk inversion statistics is defined

\section{Definition 1}

$$
\begin{aligned}
& \text { winv } N(w)=\sum_{N \in w} \# S \text { to the left of } N \\
& \text { winv } W(w)=\sum_{W \in w} \# S+\# N+\# E \text { to the left of } W \\
& \text { winv } E(w)=\sum_{E \in w} \# S+\# N+2 \# W \text { to the left of } E \\
& \text { wpinv }(w)=\operatorname{winv} N(w)+\operatorname{winv} W(w)+\operatorname{winv} E(w)
\end{aligned}
$$

Example 3 Here are the inversions of the walks (from left to right) on Fig. 1 .

$$
\begin{aligned}
& W N E N=2 \cdot 1+1=3 \\
& E N W N=1+1=2 \\
& N W N E=1+(2+2 \cdot 1)=5 \\
& N E N W=1+(2+1)=4 \\
& W N N E=2+2 \cdot 1=4 \\
& E N N W=1+2=3
\end{aligned}
$$




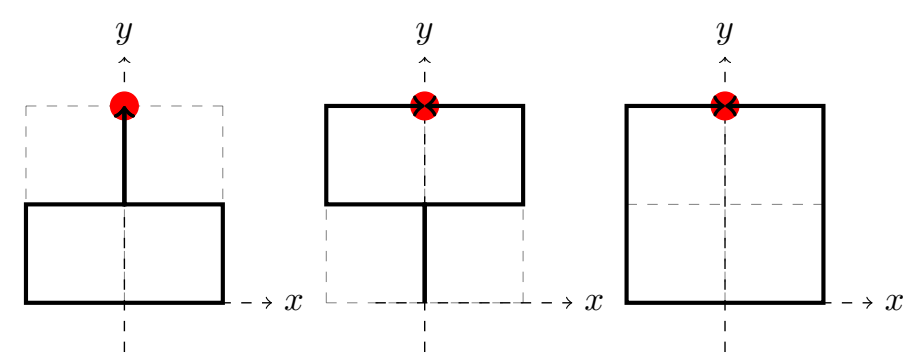

Fig. 1: Some walks from $(0,0)$ to $(0,2)$ with $n=4$ steps.

Denote the set of all lattice walks from $(a, b)$ to $(c, d)$ in $n$ letters (steps) by $P_{n}((a, b) \mid(c, d))$. The $q$-analog of the walk enumeration formula is the following generating function.

\section{Proposition 1}

$$
\left[\begin{array}{c}
n \\
\frac{1}{2}(n-c+d)
\end{array}\right]_{q}\left[\begin{array}{c}
n \\
\frac{1}{2}(n-c-d)
\end{array}\right]_{q}=\sum_{w \in P_{n}((0,0) \mid(c, d))} q^{w i n v(w)}
$$

The following is true for the walks restricted to the upper half plane. Denote the set of walks starting at $(0,0)$ and ending at $(c, d)$ in $n$ steps and never going below the $x$-axis by $P_{n}^{+}((0,0) \mid(c, d))$, then

\section{Proposition 2}

$$
\begin{aligned}
{\left[\begin{array}{c}
n \\
\frac{1}{2}(n+c+d)
\end{array}\right]_{q}\left[\begin{array}{c}
n \\
\frac{1}{2}(n+c-d)
\end{array}\right]_{q}-q^{d+1}\left[\begin{array}{c}
n \\
\frac{1}{2}(n+c+d)+1
\end{array}\right]_{q}\left[\begin{array}{c}
n \\
\frac{1}{2}(n+c-d)-1
\end{array}\right]_{q} } & =\sum_{w \in P_{n}^{+}((0,0) \mid(c, d))} q^{\operatorname{winv}(w)}
\end{aligned}
$$

In Section 6I will also introduce an analog of a major index, $w \operatorname{maj}(w)$, which is, conjecturally, equally distributed with $\operatorname{winv}(w)$ over lattice walks.

\section{$3 q$-Lassalle Numbers}

The purpose of this section is to derive a bilinear recursion relations for $A_{k}(q)$ and $\alpha_{k}(q)$, from which the positivity and integrality follow.

The strategy is to rewrite the recursion relation between $A^{\prime}(q)$ (as in (1)) as a difference equation. Then, using the q-difference equation for the generating function of $q$-Catalan, to obtain the $q$-difference equation for the generating function of the $q$-Lassalle numbers.

$$
\begin{aligned}
& A_{n}^{\prime}(q)=(-1)^{n-1} q^{n-1} C_{n}(q)+\sum_{j=1}^{n-1}(-1)^{n-j-1} q^{n-j}\left[\begin{array}{c}
2 n-1 \\
2 j-1
\end{array}\right]_{q} A_{j}^{\prime}(q) C_{n-j}(q) \\
& -\frac{(-1)^{n} q^{n}[2 n]_{q} C_{n}(q)}{[2 n]_{q} !}=\frac{q A_{n}^{\prime}(q)}{[2 n-1]_{q} !}+\sum_{j=1}^{n-1} \frac{q A_{j}^{\prime}(q)}{[2 j-1]_{q} !} \frac{(-1)^{n-j} q^{n-j} C_{n-j}(q)}{[2 n-2 j]_{q} !}
\end{aligned}
$$


Introduce generating functions and the finite $q$-difference operator:

$$
\begin{aligned}
H(t ; q) & =\sum_{k \geq 0} \frac{(-1)^{k} q^{k} C_{k}(q) t^{2 k}}{[2 k]_{q} !} \equiv \sum_{k \geq 0} \frac{(-1)^{k} q^{k} t^{2 k}}{[k]_{q} ![k+1]_{q} !} \\
P(t ; q) & =\sum_{k \geq 1} \frac{q A_{k}^{\prime}(q) t^{2 k-1}}{[2 k-1]_{q} !} \\
D_{q} f(t) & =\frac{f(t)-f(q t)}{(1-q) t}
\end{aligned}
$$

then (7) is equivalent to

$$
-D_{q} H(t, q)=P(t ; q) H(t ; q)
$$

Recall Jackson's basic $q$-Bessel function ([Ism82] $)$ :

$$
J_{\nu}^{(1)}(x ; q)=\frac{\left(q^{\nu+1} ; q\right)_{\infty}}{(q ; q)_{\infty}} \sum_{n \geq 0} \frac{(-1)^{n}}{(q ; q)_{n}\left(q^{\nu+1} ; q\right)_{n}}\left(\frac{x}{2}\right)^{2 n+\nu}, \quad \text { where } \quad(a ; q)_{n}=\prod_{j=0}^{n-1}\left(1-a q^{i}\right)
$$

For $\nu=1$

$$
J_{1}^{(1)}(x ; q)=\frac{1}{1-q} \sum_{n \geq 0} \frac{(-1)^{n}}{(q ; q)_{n}\left(q^{2} ; q\right)_{n}}\left(\frac{x}{2}\right)^{2 n+1}=\sum_{n \geq 0} \frac{(-1)^{n}}{[n]_{q} ![n+1]_{q} !}\left(\frac{x}{2(1-q)}\right)^{2 n+1}
$$

So that

$$
H(t ; q)=\frac{1}{\sqrt{q} t} J_{1}^{(1)}(2(1-q) \sqrt{q} t ; q), i . e .
$$

$q$-Bessel function $J_{1}^{(1)}(x ; q)$ is a generating function for $q$-Catalan numbers.

$J_{1}^{(1)}(x ; q)$ satisfies the following $q$-Bessel difference equation:

$$
J_{1}^{(1)}(q x ; q)-\left(q^{\frac{1}{2}}+q^{-\frac{1}{2}}\right) J_{1}^{(1)}(\sqrt{q} x ; q)+\left(1+\frac{x^{2}}{4}\right) J_{1}^{(1)}(x ; q)=0
$$

For $H(t ; q), 10)$ translates to:

$$
H(q t ; q)-\left(1+\frac{1}{q}\right) H(\sqrt{q} t ; q)+\left(\frac{1}{q}+(1-q)^{2} t^{2}\right) H(t ; q)=0
$$

Through the $q$-difference equation $[8$ this implies the following ungainly looking $q$-difference equation for $P(t ; q)$ :

$$
\begin{aligned}
& \left(1+q+q(1-q)^{2} t^{2}\right) q(1-q) \sqrt{q} t P(\sqrt{q} t ; q)+q^{2}(1-q)^{2} t P(t ; q) \sqrt{q} t P(\sqrt{q} t ; q)+ \\
& +\left(1+q+q(1-q)^{2} t^{2}\right) q^{2}(1-q)^{2} t^{2}+q^{2}(1-q)^{2} t^{2} q(1-q) t P(t ; q) \\
& =\left(1-q^{2}\right) t P(t ; q)-q(1-q)\left(1-q^{2}\right) t^{2}
\end{aligned}
$$


But collecting coefficients of $t^{2 n}$ on both sides makes things look better:

$$
\begin{aligned}
& A_{1}^{\prime}(q)=1 \\
& A_{2}^{\prime}(q)=q^{2} \\
& \frac{[2]_{q}[s+1]_{q}}{[2 s-1]_{q} !} A_{s}^{\prime}(q)=\frac{q^{3}+q^{s+1}}{[2 s-3]_{q} !} A_{s-1}^{\prime}(q)+\sum_{k=2}^{s-2} \frac{q^{k+3} A_{k}^{\prime}(q) A_{s-k}^{\prime}(q)}{[2 k-1]_{q} ![2 s-2 k-1]_{q} !}
\end{aligned}
$$

It's time to rescale, so let

$$
A_{k}^{\prime}(q)=q^{3 k-4} A_{k}(q)
$$

then the recursion relation for $A_{k}(q)$ is:

$$
A_{s}(q)=\frac{[2 s-1]_{q}}{[2]_{q}[s+1]_{q}} \sum_{k=1}^{s-1} q^{k-1} \frac{[2 s-2]_{q} !}{[2 k-1]_{q} ![2 s-2 k-1]_{q} !} A_{k}(q) A_{s-k}(q)
$$

Translating this recursion into that for $\alpha_{n}(q)$ (as in (3)) produces:

$$
\alpha_{n}(q)=\frac{1}{[2]_{q}[n]_{q}} \sum_{k=1}^{n-1} q^{k-1}\left[\begin{array}{c}
n \\
k+1
\end{array}\right]_{q}\left[\begin{array}{c}
n \\
k-1
\end{array}\right]_{q} \alpha_{k}(q) \alpha_{n-k}(q)
$$

The ratio of the $q$-binomial coefficients can be rewritten as

$$
\frac{1}{[n]_{q}}\left[\begin{array}{c}
n \\
k+1
\end{array}\right]_{q}\left[\begin{array}{c}
n \\
k-1
\end{array}\right]_{q}=\frac{1}{[2]_{q}}\left(\left[\begin{array}{c}
n-1 \\
r-1
\end{array}\right]_{q}\left[\begin{array}{c}
n-1 \\
r
\end{array}\right]_{q}-q^{2}\left[\begin{array}{c}
n-1 \\
r-2
\end{array}\right]_{q}\left[\begin{array}{c}
n-1 \\
r+1
\end{array}\right]_{q}\right)
$$

Therefore, the proof depends on

- positivity and integrality of

$$
c_{n, k}(q)=\left[\begin{array}{l}
n-1 \\
r-1
\end{array}\right]_{q}\left[\begin{array}{c}
n-1 \\
k
\end{array}\right]_{q}-q^{2}\left[\begin{array}{l}
n-1 \\
k-2
\end{array}\right]_{q}\left[\begin{array}{l}
n-1 \\
k+1
\end{array}\right]_{q}
$$

- divisibility of $c_{n, k}(q)$ and $\alpha_{k}(q)$ by powers of $[2]_{q}$.

As may be seen from (6), the positivity and integrality of $c_{n, k}(q)$ follows from its combinatorial interpretation as the generating function of winv statistics of upper half-plane lattice walks, namely the walks that start at $(0,0)$ and end up at $(2 k-n-1,1)$ in $(n-1)$ steps.

Therefore I continue with the lattice walk part of the story.

\section{Walk Inversion Generating Function}

In order to prove the walk inversion generating function formula, as in (5)

$$
\left[\begin{array}{c}
n \\
\frac{1}{2}(n-c+d)
\end{array}\right]_{q}\left[\begin{array}{c}
n \\
\frac{1}{2}(n-c-d)
\end{array}\right]_{q}=\sum_{w \in P_{n}((0,0) \mid(c, d))} q^{w i n v(w)}
$$


I will need the following lemma. Denote the set of all walks of length $n$ from $(0,0)$ to $(c, d)$ with exactly $k \mathrm{~W}$ steps by $P_{n}((c, d) ; k)$. Of course this fixes the number of $\mathrm{S}$ steps, $r$ as well. The total number of steps in $n,(c+k)$ of which are $\mathrm{E}, k-\mathrm{W},(d+r)-\mathrm{N}$, and $r-\mathrm{S}$.

$$
r=\frac{1}{2}(n-c-d)-k
$$

As words, these walks are permutations of each other. Their total number is $\frac{n !}{(c+k) ! k !(d+r) ! r !}$.

Lemma 1 The generating function of the walk inversion statistics of $n$-step walks with $k$ W steps is given by

$$
q^{k^{2}+c k} \frac{[n]_{q} !}{[c+k]_{q} !\left[\frac{1}{2}(n-c+d)-k\right]_{q} ![k]_{q} !\left[\frac{1}{2}(n-c-d)-k\right]_{q} !}=\sum_{w \in P_{n}((c, d) ; k)} q^{\text {winv }(w)}
$$

Now, in general, to get to $(c, d)$ from $(0,0)$ in $n$ steps one might take just one $\mathrm{W}$ step, or two, etc. The maximal number of $\mathrm{W}$ steps (i.e. no $\mathrm{S}$ steps) is

$$
\frac{1}{2}(n-c-d)
$$

So that

Lemma 2 The walk inversion generating function is

$$
\sum_{w \in P_{n}((0,0) \mid(c, d))} q^{w i n v(w)}=\sum_{k=0}^{\frac{1}{2}(n-c-d)} q^{k^{2}+c k} \frac{[n]_{q} !}{[c+k]_{q} !\left[\frac{1}{2}(n-c+d)-k\right]_{q} ![k]_{q} !\left[\frac{1}{2}(n-c-d)-k\right]_{q} !}
$$

Now, consider how a walk can end up at $(c, d)$ in $n$ steps, i.e. where could it be at the previous step?

- either at $(c-1, d)$, with the last step E; adding an E step changes winv by $\# S+\# N+2 \# W=$ $r+(d+r)+2 k=d+2 r+2 k=n-c$

The contribution of walks coming from the West is

$$
q^{n-c} \sum_{k=0}^{\frac{1}{2}(n-c-d)} q^{k^{2}+(c-1) k} \frac{[n-1]_{q} !}{[c-1+k]_{q} !\left[\frac{1}{2}(n-c-d)-k\right]_{q} ![k]_{q} !\left[\frac{1}{2}(n-c+d)-k\right]_{q} !}
$$

- or at $(c, d+1)$ with the last step $\mathrm{S}$; adding an $\mathrm{S}$ step does not change winv.

The contribution of the walks coming from the North:

$$
\sum_{k=0}^{\frac{1}{2}(n-c-d)-1} q^{k^{2}+c k} \frac{[n-1]_{q} !}{[c+k]_{q} !\left[\frac{1}{2}(n-c+d)-k-1\right]_{q} ![k]_{q} !\left[\frac{1}{2}(n-c-d)-k-1\right]_{q} !}
$$


- or at $(c+1, d)$ with the last step $\mathrm{W}$; adding a $\mathrm{W}$ step changes winv by $\# S+\# N+\# E=$ $r+(d+r)+(k+c+1)=d+2 r+c+k+1=n-1-k$

The contribution of walks coming from the East is

$$
q^{n-1} \sum_{k=0}^{\frac{1}{2}(n-c-d)-1} q^{k^{2}+c k} \frac{[n-1]_{q} !}{[c+1+k]_{q} !\left[\frac{1}{2}(n-c+d)-k-1\right]_{q} ![k]_{q} !\left[\frac{1}{2}(n-c-d)-k-1\right]_{q} !}
$$

- or at $(c, d-1)$ with the last step $\mathrm{N}$; an addition of an $\mathrm{N}$ step with $r$ prior $\mathrm{S}$ steps changes winv by $\frac{1}{2}(n-c-d)-k$.

The contribution of the walks coming from the South:

$$
q^{\frac{1}{2}(n-c-d)} \sum_{k=0}^{\frac{1}{2}(n-c-d)} q^{k^{2}+(c-1) k} \frac{[n-1]_{q} !}{[c+k]_{q} !\left[\frac{1}{2}(n-c-d)-k\right]_{q} ![k]_{q} !\left[\frac{1}{2}(n-c+d)-k\right]_{q} !}
$$

The sum of these contributions gives a recursion that establishes 5 .

\section{Upper Half-Plane walks}

Following the logic of walks reflection [GKS92], to every negative path from $(0,0)$ to $(c, d)$ will associate a walk from $(-2,0)$ to $(c, d)$ so that the change in winv is the same for every walk.

Here is the algorithm.

1. Separate each negative walk in two segments $w=w_{1} \cdot w_{2}$, where $\cdot$ means concatenation of words:

(a) $w_{1}$ : the part of the walk that starts at $(0,0)$ and ends at $(*, 0)$ (before it dips below the $x$-axis the first time)

(b) $w_{2}$ : the rest of the walk that runs from $(*, 0)$ to $(c, d)$; Notice that $w_{2}$ starts with $\mathbf{S}$ and necessarily has at least $d+1 \mathrm{~N}$ steps. More precisely, if the walk has $k \mathrm{~S}$ steps, it has $d+k$ N steps.

2. $\tilde{w}_{1}$ : move $w_{1}$ down two steps, so that it starts at $(0,-2)$ and ends at $(*,-2)$;

3. attach $\tilde{w}_{2}$ to the combined walk. The walk modified this way runs from $(0,-2)$ to $(c, d)$

Consider the simplest (negative) walk $w=w_{1} \cdot w_{2}$ : after dipping down below the $x$-axis it goes straight up to $d$, i.e. the part after reaching the $x$-axis $\left(w_{2}\right)$ looks like

$$
w_{2}=S \underbrace{N N \ldots N N}_{d+1 \text { times }}
$$

Transform this walk into

$$
\tilde{w}_{2}=\underbrace{N N \ldots N N}_{d+1 \text { times }} S
$$

Suppose that $w_{1}$ contained $k \mathrm{~S}$ steps. Then $\left(\tilde{w}=\tilde{w}_{1} \cdot \tilde{w}_{2}\right)$

$$
\operatorname{winv}(w)-\operatorname{winv}(\tilde{w})=(d+1)(k+1)-(d+1) k=d+1
$$


Now consider a generic $w$ with blocks of $\mathrm{S}$ and $\mathrm{N}$ letters:

$$
w_{2}=\underbrace{S \ldots S}_{s_{1} \text { times }} \underbrace{N \ldots N}_{n_{1} \text { times }} \underbrace{S \ldots S}_{s_{2} \text { times }} \underbrace{N \ldots N}_{n_{2} \text { times }} \cdots \underbrace{S \ldots S}_{s_{r} \text { times }} \underbrace{N \ldots N}_{n_{r} \text { times }}
$$

where

$$
\sum_{i}^{r} n_{i}-s_{i}=d
$$

By swapping the last $\mathrm{S}$ in the each string of $s_{i}$ with the last $\mathrm{N}$ letter in $n_{i}$ until $d+1$ letters $\mathrm{N}$ have been moved, transforms $w_{2}$

$$
\begin{aligned}
& \tilde{w}_{2}=\underbrace{S \ldots S}_{s_{1}-1 \text { times }} \underbrace{N N \ldots N N}_{n_{1} \text { times }} S \underbrace{S \ldots S}_{s_{2}-1 \text { times }} \underbrace{N \ldots N}_{n_{2} \text { times }} S \ldots \underbrace{S \ldots S}_{s_{i}-1 \text { times }} \underbrace{N \ldots N}_{r \text { times }} S \underbrace{N \ldots N}_{n_{i}-r \text { times }} \ldots \underbrace{S \ldots S}_{s_{r} \text { times }} \underbrace{N \ldots N}_{n_{r} \text { times }} \\
& \text { with } n_{1}+n_{2}+\ldots+r=d+1 \\
& \text { winv }(w)-\operatorname{winv}(\tilde{w})=\left\{n_{1}\left(s_{1}+k\right)-n_{1}\left(s_{1}-1+k\right)\right\}+\left\{n_{2}\left(s_{1}+s_{2}+k\right)-n_{2}\left(s_{1}+s_{2}-1+k\right)\right\} \\
& +\ldots+\left\{r\left(s_{1}+\ldots+s_{i}+k\right)-r\left(s_{1}+\ldots+s_{i}-1+k\right)\right\} \\
& +\left\{\left(n_{i}-r\right)\left(s_{1}+\ldots+s_{i}+k\right)-\left(n_{i}-r\right)\left(s_{1}+\ldots+s_{i}+k\right)\right\}+ \\
& +\ldots+\left\{n_{r}\left(s_{1}+\ldots+s_{r}\right)-n_{r}\left(s_{1}+\ldots+s_{r}\right)\right\}=\sum_{i} n_{i}+r=d+1 \\
& \operatorname{winv}(\tilde{w})=q^{-(d+1)} \operatorname{winv}(w)
\end{aligned}
$$

So the total contribution of negative walks is

$$
q^{d+1} P_{n}((0,-2) \mid(c, d))=q^{d+1}\left[\begin{array}{c}
n \\
\frac{1}{2}(n+2+c+d)
\end{array}\right]_{q}\left[\begin{array}{c}
n \\
\frac{1}{2}(n-2+c-d)
\end{array}\right]_{q}
$$

Hence (6).

\section{Major Walk Index}

Setting up the following order $S>N>E>W$, and with the usual definition of a descent set

$$
\begin{aligned}
& \operatorname{des} N(w)=\left\{i: \mathrm{S} \text { occurs as } i^{\text {th }} \text { letter and N occurs as } i+1^{\text {st }} \text { letter }\right\} \\
& \operatorname{maj} N(w)=\sum_{i \in \operatorname{des} N(w)} i \\
& \operatorname{des} E(w)=\left\{i: \mathrm{S} \text { or } \mathrm{N} \text { occur as } i^{\text {th }} \text { letter and E occurs as } i+1^{\text {st }} \text { letter }\right\} \\
& \operatorname{maj} E(w)=\sum_{i \in \operatorname{des} E(w)} i \\
& \operatorname{des} W(w)=\left\{i: \mathrm{S}, \mathrm{N}, \text { or E occur as } i^{\text {th }} \text { letter and } \mathrm{W} \text { occurs as } i+1^{\text {st }} \text { letter }\right\} \\
& \operatorname{maj} W(w)=\sum_{i \in \operatorname{des} W(w)} i
\end{aligned}
$$

one can give the following 
Definition 2

$$
w \operatorname{maj}(w)=\operatorname{maj} N(w)+\operatorname{maj} E(w)+\operatorname{maj} W(w)+\# E \times \# W
$$

Notice the unusual last term... Nevertheless, experiments show that winv and wmaj are equally distributed over all lattice walks as well as over upper half-plane walks.

\section{Conjecture 1}

$$
\sum_{w \in P_{n}((0,0) \mid(c, d))} q^{\operatorname{winv}(w)}=\sum_{w \in P_{n}((0,0) \mid(c, d))} q^{\operatorname{wmaj}(w)}
$$

$$
\sum_{w \in P_{n}^{+}((0,0) \mid(c, d))} q^{\operatorname{winv}(w)}=\sum_{w \in P_{n}^{+}((0,0) \mid(c, d))} q^{\operatorname{wmaj}(w)}
$$

\section{$7 q$-Integers Associated with $q$-super Ballot Numbers}

Computer experiments indicate that there is a family of $q$-numbers related to several generalizations of $q$-Catalan. For instance, following [Ges92] define $q$-super Ballot numbers

$$
B_{n, k, r}(q)=\frac{[k+2 r]_{q} ![2 n+k-1]_{q} !}{(k-1)_{q} ! r_{q} ! n_{q} ![n+k+r]_{q} !}
$$

Then, I venture to make the following conjecture

Conjecture 2 Define a new sequence $A_{n, k, r}(q)$ with $A_{1, k, r}=B_{0, k, r}(q)$ through the recurrence

$$
(-1)^{n-1} A_{n, k, r}(q)=q^{n-1} B_{n, k, r}(q)+\sum_{j=1}^{n-1}(-1)^{j} q^{n-j-1}\left[\begin{array}{c}
2 n-1 \\
2 j-1
\end{array}\right]_{q} A_{j, k, r}(q) B_{n-j, k, r}(q)
$$

then the $A_{n, k, r}(q)$ are polynomials in $q$ with positive integers coefficients for all values of $n, k, r>0$.

\section{Example 4}

$$
\begin{aligned}
A_{2,1,1}(q)= & 1+2 q+4 q^{2}+4 q^{3}+3 q^{4}+q^{5} \\
A_{2,2,1}(q)= & +3 q+7 q^{2}+11 q^{3}+13 q^{4}+12 q^{5}+8 q^{6}+4 q^{7}+q^{8} \\
A_{2,2,2}(q)= & +3 q+9 q^{2}+18 q^{3}+33 q^{4}+51 q^{5}+72 q^{6}+89 q^{7}+100 q^{8}+101 q^{9}+93 q^{10}+77 q^{11} \\
& +57 q^{12}+38 q^{13}+22 q^{14}+11 q^{15}+4 q^{16}+q^{17} \\
A_{3,1,1}(q)= & +3 q+9 q^{2}+17 q^{3}+28 q^{4}+38 q^{5}+44 q^{6}+43 q^{7}+35 q^{8}+24 q^{9}+13 q^{10}+5 q^{11}+q^{12} \\
A_{3,2,1}(q)= & +5 q+19 q^{2}+51 q^{3}+110 q^{4}+199 q^{5}+307 q^{6}+412 q^{7}+484 q^{8}+499 q^{9}+452 q^{10} \\
& +358 q^{11}+245 q^{12}+143 q^{13}+69 q^{14}+26 q^{15}+7 q^{16}+q^{17} \\
A_{3,2,2}(q)= & +5 q+22 q^{2}+68 q^{3}+181 q^{4}+414 q^{5}+848 q^{6}+1567 q^{7}+2652 q^{8}+4134 q^{9}+5980 q^{10} \\
& +8058 q^{11}+10155 q^{12}+11997 q^{13}+13313 q^{14}+13892 q^{15}+13639 q^{16}+12597 q^{17}+ \\
& +10937 q^{18}+8913 q^{19}+6802 q^{20}+4845 q^{21}+3206 q^{22}+1958 q^{23}+1094 q^{24} \\
& ++552 q^{25}+247 q^{26}+95 q^{27}+30 q^{28}+7 q^{29}+q^{30}
\end{aligned}
$$




\section{Acknowledgements}

I would like to thank Jean-Christophe Novelli and Jean-Yves Thibon for an interesting and useful conversation.

\section{References}

[DR84] D. DeTemple and J. Robertson. Equally likely fixed length paths in graphs. Ars Comb., 17:243$254,1984$.

[Ges92] I. Gessel. Super ballot numbers. 1992.

[GKS92] R. K. Guy, C. Krattenthaler, and B.E. Sagan. Lattice paths, reflections, and dimension-changing bijections. Ars Comb., 34:3-15, 1992.

[Ism82] M.E.H. Ismail. The zeros of basic bessel functions, the functions $j_{\nu+a x}(x)$, and associated orthogonal polynomials. Journal of Mathematical Analysis and Applications, 86(1-19), 1982.

[Las12] M. Lassalle. Two integer sequences related to Catalan numbers. Journal of Combinatorial Theory, Series A, 119:923-935, 2012. 
